\title{
Complicaciones infecciosas asociadas a la nefrolitotomía percutánea en un hospital universitario de Colombia
}

\section{Infectious Complications Associated to Percutaneous Nephrolithotomy in a Teaching Hospital from Colombia}

\author{
Adriana María Jiménez ${ }^{1 *}$ Diego Armando Riatiga Ibañez ${ }^{2} \quad$ Armando Sánchez López ${ }^{2}$ \\ José Daniel Daza Pupo ${ }^{3}$ \\ 1 Profesor Asociado de la Facultad de Medicina de la Fundación \\ Universitaria de Ciencias de la Salud. Coordinadora de la Unidad de \\ Prevención y Control de Infecciones del Hospital de San José, Bogotá, \\ Colombia \\ 2 Instructor Asistente de la Facultad de Medicina de la Fundación

\begin{abstract}
Address for correspondence Adriana María Jiménez, MD, Profesor Asociado de la Facultad de Medicina de la Fundación Universitaria de Ciencias de la Salud., Coordinadora de la Unidad de Prevención y Control de Infecciones del Hospital de San José, Cra 19 No. 8A - 32,
\end{abstract} \\ Bogotá, Colombia (e-mail: amjimenez@fucsalud.edu.co).
} Universitaria de Ciencias de la Salud. Servicio de Urología del Hospital de San José, Bogotá, Colombia

3 Residentes de IV año del Postgrado de Urología. Fundación

Universitaria de Ciencias de la Salud, Bogotá, Colombia

Urol Colomb 2019;28:149-153.

\section{Resumen}

\section{Palabras clave}

- complicaciones infecciosas

- nefrolitotomia percutánea

- cálculos urinarios

- sepsis

- profilaxis antibiótica
Introducción La Nefrolitotomía Percutánea (NLP), es una técnica mínimamente invasiva empleada para el manejo de los cálculos de gran tamaño. Diferentes tipos de complicaciones se pueden presentar después de ese procedimiento. El propósito de este estudio es establecer la frecuencia y características asociadas, de las complicaciones infecciosas de los pacientes sometidos a NLP en un hospital universitario de cuarto nivel de atención que es un centro de remisión de pacientes con patología urológica.

Materiales y Métodos Estudio descriptivo, retrospectivo que evalúa 164 pacientes sometidos a NLP en el Hospital Universitario de San José de Bogotá (Colombia) desde enero 2010 hasta diciembre del 2014. Se buscó la incidencia de cualquier tipo de complicación infecciosa durante los 30 días del post operatorio. Se recolectó información concerniente a los antecedentes de hospitalización, uso de antibióticos, infección del tracto urinario, características del cálculo, profilaxis empleada, cultivos realizados, patrón de sensibilidad, necesidad de cambio de antibiótico y evolución.

Resultados Se incluyeron 164 pacientes, de los cuales $12,8 \%$ presentaron complicaciones infecciosas. El 95\% de los pacientes pertenecían al sexo femenino, la infección más frecuente fue la de la vía urinaria (86\%) y el microorganismo identificado con mayor frecuencia fue Escherichia coli que presentó solo un $42 \%$ de sensibilidad a la cefazolina. El $61,90 \%$ (13) de los pacientes presentaron sepsis, encontrando una mayor frecuencia en aquellos con antecedentes de: infección de vías urinaria (84,62\%), hospitalización por cirugía $(46,12 \%)$, cálculos $>25$ mm $(92,31 \%)$ y densidad $>1000$ UH $(38,46 \%)$. received

September 10, 2018

accepted

February 14, 2019
DOI https://doi.org/

$10.1055 / \mathrm{s}-0039-1683992$.

ISSN 0120-789X.

eISSN 2027-0119.
Copyright ( 2019 , Sociedad Colombiana License terms de Urología. Publicado por Thieme Revinter Publicações Ltda., Rio de Janeiro, Brazil. Todos los derechos reservados.

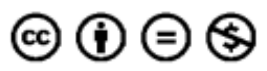




\begin{abstract}
Keywords

- infectious complications

- percutaneos nephrolitotomy

- urinary stones

- sepsis

- antibiotic prophylaxis

Introduction The NLP is a minimal invasive technique used for handling big size calculus. Different kind of complications may be presented after this procedure. The purpose of this study is to stablish the frequency and the associated features of the infectious complications of the patients who underwent NLP in a university hospital which is a reference center.

Materials and Methods A Descriptive, retrospective survey evaluating 164 patients undergoing PNL in San Jose University hospital from January 2010 to December 2014. The incidence of any type of infectious complication was sought within 30 days after surgery. Information concerning hospitalization history, use of antibiotics, urinary tract infection, stone characteristics, prophylaxis used, cultures made, sensitivity pattern, the need for antibiotic change and evolution was collected.

Results 164 patients were included, in which $12,8 \%$ of them presented infectious complications. $95 \%$ of the patients were females, urinary infection (86\%) was the most frequent among them and the most recognized microorganism was Escherichia coli, in which only $42 \%$ had cefazolin sensitivity. $61,90 \%$ (13) of the patients presented sepsis, with higher frequency on those with history of: urinary infection (84,63\%), surgery hospitalization (46,12\%), stones $>25 \mathrm{~mm}(92,31 \%)$ and density $>1000 \mathrm{UH}(38,46 \%)$ Conclusions The infections are one of the complications described after a PNL due to the destruction of infectious stones of great lithiasic mass and associated to urinary tract obstruction. To minimize this risk, the right prophylaxis must be established according to the risk factors of the patient and the local epidemiology. It is important to do stone fragments cultures with the purpose of implementing the appropriate antibiotic in case an infection is presented.
\end{abstract}

Conclusiones Las infecciones son una de las complicaciones descritas posterior a una NLP debido a la destrucción de cálculos infecciosos de gran carga litíasica y asociados a la obstrucción del tracto urinario. Con el fin de minimizar ese riesgo, se debe administrar la profilaxis adecuada establecida de acuerdo a los factores de riesgo del paciente y a la epidemiología local. Es importante realizar cultivos de los fragmentos del cálculo con el fin de instaurar el antibiótico apropiado en caso de presentarse una infección.

\section{Introducción}

En 1976 se llevó a cabo la primera Nefrolitotomía Percutánea (NLP), ${ }^{1}$ procedimiento que ha resurgido en la última década, gracias al mejoramiento de la técnica quirúrgica lo cual se ha evidenciado en la disminución en el riesgo de complicaciones y una tasa libre de cálculos $>90 \%{ }^{2}$ No existe una clasificación universal de las complicaciones secundarias a ese procedimiento, aunque en los últimos años se ha promovido el empleo de la clasificación de Clavien modificada con el fin de estandarizar su reporte.,

Los pacientes con mayor riesgo de complicaciones infecciosas presentan cálculos de tipo coraliforme, insuficiencia renal, edad avanzada, sexo femenino, derivaciones urinarias, obstrucción del tracto urinario, catéteres permanentes, acidosis tubular renal, riñón esponjoso medular, vejiga neurogénica e inmunosupresión. Los factores intraoperatorios asociados al desarrollo de Respuesta Inflamatoria Sistémica post NLP son la hidronefrosis, la carga litiásica, NLP previa ipsilateral, cálculos caliceales, tipo de cálculos, múltiples trayectos de acceso, tiempo quirúrgico, la cantidad de líquido de irrigación y las transfusiones postoperatorias. ${ }^{5-7}$

Aunque la fiebre aislada es relativamente frecuente después del procedimiento (21\%-32,1\%), la incidencia de la sepsis $(0,3 \%-4,7 \%)$ y de choque séptico $(0,3-1 \%)$ es bajo. 8,9

A pesar de que la frecuencia de complicaciones infecciosas es baja, ellas implican en un sobrecosto derivado de los tiempos de hospitalización, del uso de antibióticos y las de mayor severidad, se asocian con un alto riesgo de mortalidad.

Los estudios que describen de manera específica el tipo de infecciones posterior a NLP, los agentes etiológicos implicados y su patrón de resistencia son escasos. El objetivo de este estudio, es caracterizar las infecciones como complicación de la NLP con el fin de establecer la magnitud de ese fenómeno y contribuir al uso racional de antibióticos.

\section{Materiales y Métodos}

Se trata de un estudio descriptivo, retrospectivo, observacional, realizado entre enero de 2010 y diciembre 
2014 en el Hospital San José de Bogotá, un hospital Universitario de cuarto nivel de atención que es centro de remisión de pacientes con patología urológica. Se realizó un muestreo no probabilístico y se analizaron las historias clínicas de todos los pacientes que fueron sometidos a NLP con técnica convencional en decúbito prono en la totalidad de los pacientes; se realizó dilatación secuencial con dilatadores de Alken, usando camisas Amplatz de diámetros entre 24 y $26 \mathrm{fr}$. En la totalidad de los pacientes, se usó catéter doble Jota post operatorio y nefrostomía percutánea. El antibiótico profiláctico usado en los pacientes sometidos al estudio fue Cefazolina dosificado de acuerdo con el peso del paciente, el cual se continuó hasta el final de la hospitalización o hasta que fuese sustituido de acuerdo al resultado del urocultivo durante la hospitalización. Los pacientes incluidos en el estudio tenían urocultivo previo al procedimiento negativo. Se emplearon definiciones de infección del Center for Disease Control and Prevention de los Estados Unidos. ${ }^{10}$ Se excluyeron pacientes con historias clínicas incompletas y pacientes con antecedente de cateterismo intermitente 0 sonda vesical a permanencia ya que esos procedimientos constituyen un factor de riesgo adicional y de origen comunitario para el desarrollo de Infección del Tracto Urinario (ITU).

Se evaluaron las características demográficas edad y género, los antecedentes médicos del paciente: ITU, hospitalización en los 3 meses previos, litiasis, tratamiento previo de patología litiásica; las características del cálculo como localización, tamaño y densidad; las características de la cirugía, la profilaxis de antibiótico, la presencia de SIRS y los resultados de los cultivos y el antibiograma.

Se realizó análisis descriptivo de los datos empleando el programa Stata $12 \AA$; se utilizaron frecuencias absolutas, relativas, y medidas de tendencia central y de dispersión, como indicadores estadísticos.

El protocolo de investigación contó con el aval del Comité de Ética del Hospital San José.

\section{Resultados}

El total de pacientes incluidos en la investigación fue de 164 con una tasa de complicaciones infecciosas global del 12,8\% (21 pacientes), siendo la infección más frecuente la ITU $(85,7 \%)$, seguido por bacteriemia $(9,5 \%)$ y neumonía $(4,8 \%)$; solo uno de los pacientes presentó dos infecciones (ITU más infección del sitio operatorio-ISO-). La - Tabla 1 resume las variables demográficas y clínicas de los pacientes que presentaron la complicación infecciosa.

Como antecedentes de importancia, la mayoría pertenecía al sexo femenino (95,2\%); tenían ITU previa (71,43\%) e hipertensión como comorbilidad (43\%). En cuanto a las características del cálculo, en la mayoría se documentó cálculos con tamaño mayor a $25 \mathrm{~mm}$ (86\%) y localizados en la pelvis renal (48\%).

La fiebre fue el signo de la Respuesta Inflamatoria Sistémica (SIRS) más frecuente, registrándose en el 71\% de los pacientes infectados; en $20 \%$ de ellos de manera aislada y
Tabla 1 Características demográficas y clínicas de los pacientes con complicación infecciosa

\begin{tabular}{|c|c|c|}
\hline & $n$ & $\%$ \\
\hline Edad (promedio, DE) & 42,04 & 9,4 \\
\hline $\begin{array}{l}\text { Tiempo Quirúrgico } \\
\text { (minutos promedio, rango) }\end{array}$ & 120 & $(75-328)$ \\
\hline $\begin{array}{l}\text { Estancia Hospitalaria } \\
\text { (días promedio, rango) }\end{array}$ & 4,85 & $(2-9)$ \\
\hline \multicolumn{3}{|l|}{ Sexo } \\
\hline Masculino & 1 & 4,8 \\
\hline Femenino & 20 & 95,2 \\
\hline \multicolumn{3}{|l|}{ Manejo previo de litiasis } \\
\hline Sí & 9 & 43 \\
\hline No & 12 & 57 \\
\hline \multicolumn{3}{|l|}{ Antecedente de IVU } \\
\hline Sí & 15 & 71 \\
\hline No & 6 & 29 \\
\hline \multicolumn{3}{|l|}{ Comorbilidades } \\
\hline Hipertensión & 9 & 42,9 \\
\hline Diabetes & 4 & 19,1 \\
\hline Inmunosupresión & 0 & 0 \\
\hline OTROS & 10 & 47,6 \\
\hline \multicolumn{3}{|l|}{ Tamaño del cálculo en mm } \\
\hline $15-20 \mathrm{~mm}$ & 1 & 4,8 \\
\hline $20-25 \mathrm{~mm}$ & 2 & 9,5 \\
\hline mayor a $25 \mathrm{~mm}$ & 18 & 85,7 \\
\hline \multicolumn{3}{|l|}{ Densidad en UH } \\
\hline $500-1000$ & 3 & 14,3 \\
\hline mayor a 1000 & 7 & 33,3 \\
\hline No reportado & 11 & 52,4 \\
\hline \multicolumn{3}{|l|}{ Localización del cálculo } \\
\hline Pelvis renal & 10 & 47,6 \\
\hline Completo & 8 & 28,1 \\
\hline Superior & 6 & 28,6 \\
\hline Inferior & 5 & 23,8 \\
\hline Positivos & 2 & 9,5 \\
\hline Negativos & 19 & 90,5 \\
\hline \multicolumn{3}{|l|}{ Uso de Hemoderivados } \\
\hline Sí & 0 & 0 \\
\hline No & 21 & 100 \\
\hline \multicolumn{3}{|l|}{ Escalonamiento Antibiótico } \\
\hline Sí & 14 & 66,7 \\
\hline No & 7 & 33,3 \\
\hline
\end{tabular}

en el $80 \%$ asociado a otro signo de SIRS. El $47 \%$ de los pacientes presentaron leucocitosis; $61 \%$ de los pacientes reunieron los criterios de sepsis, presentándose con mayor frecuencia en aquellos con antecedente de ITU previas, 
hospitalización previa por cirugía, cálculos $>25 \mathrm{~mm}$ y densidad $>1000 \mathrm{UH}$. Un paciente presentó shock séptico y requirió manejo en la Unidad de Cuidados Intensivos.

En relación al uso de antibióticos, todos los pacientes contaron con profilaxis antibiótica y el $28,5 \%$ de los pacientes tenían como antecedente, haber recibido antibióticos orales previo al procedimiento quirúrgico. En el $66 \%$ de los pacientes se debió escalar el antibiótico de acuerdo a los resultados de los cultivos. El sitio de acceso renal fue cáliz inferior (80,95\%) y cáliz medio (19,05\%). El tiempo quirúrgico presentó una mediana de 120 minutos con un rango de 75 a 328 minutos. Ningún paciente requirió transfusión posoperatoria. En promedio los pacientes presentaron una hospitalización posquirúrgica de 4,85 ( \pm 2 2,2) días, mínima de 2 y máxima de 9 días. No se presentaron complicaciones intraoperatorias.

Se realizó el urocultivo en un 95\% de los pacientes, con recuperación de microorganismo en el 90\% de ellos. En el 90\% de los cultivos, se identificaron Gram negativos, siendo la bacteria aislada con más frecuencia la Escherichia coli. El 33\% de las E.coli presentaron un patrón de Beta lactamasa de Espectro Extendido.

En la - Tabla 2 se presenta la frecuencia de las bacterias identificadas.

Se realizó hemocultivo a todos los pacientes que reunieron los criterios de sepsis, pero solo en dos pacientes (16\%) resultaron positivos, un caso con aislamiento de E. coli y en el otro Staphylococcus epidermidis. El cultivo del único paciente que presentó infección del sitio operatorio arrojó un resultado polimicrobiano (Serratia marcescens, Proteus mirabilis, Pseudomonas aeruginosa).

\section{Discusión}

Las complicaciones infecciosas post NLP son esperadas dado que las indicaciones de ese procedimiento incluyen cálculos de gran tamaño que con frecuencia están colonizados por microorganismos. La incidencia de infecciones post NLP ha sido establecida entre $2,8 \%$ y $32,1 \%,{ }^{11}$ y en esta serie fue del $12,8 \%$.

La fiebre como signo aislado (Clavien Grado I) se presentó como complicación en el 1,8\% de los casos. Esa incidencia es inferior a lo encontrado en otros estudios como el de Lee que lo encontró en el $22 \%$ de 582 pacientes $^{12}$; Dogan en $23,6 \%$ de $338^{13}$; Tefleki $^{14}(2,8 \%)$ en una serie de 811 NLP y el estudio multicéntrico de Gutierrez ${ }^{15}$ y colaboradores, que incluye la que tal vez sea la serie más grande de pacientes, identificó que

Tabla 2 Microorganismos identificados en urocultivo

\begin{tabular}{|l|l|}
\hline & $\%$ \\
\hline Escherichia coli & 50 \\
\hline Proteus mirabilis & 11,1 \\
\hline Pseudomonas aeruginosa & 11,1 \\
\hline Serratia marcescens & 11,1 \\
\hline Enterobacter aerogenes & 5,5 \\
\hline Streptococcus pyogenes & 5,5 \\
\hline Streptococcus agalactiae & 5,5 \\
\hline
\end{tabular}

el $10 \%$ de 5313 pacientes presentaron fiebre aunque en la mayoría de esos estudios no se especifica si la fiebre se presentó como signo aislado o asociado a otra infección. La incidencia de la sepsis como complicación infecciosa (Clavien Grado II) ha sido reportada entre $9 \%$ y $37 \%$ y la sepsis severa y el shock séptico (Clavien Grado IV) entre $0,3 \%$ y $4,7 \% .{ }^{16}$ En esa serie, la incidencia de sepsis $(7,9 \%)$ y de shock séptico $(0,6 \%)$ se encuentra en el rango inferior descrito. En este estudio, la incidencia de complicaciones infecciosas (ITU, ISO, bacteremia) fue del 12,8\% que se clasifican en el Grado II de Clavien, y ese porcentaje es superior al descrito en otras series como las de $\operatorname{Dogan}^{13}$ (bacteriuria $8 \%$ ) y Tefleki $^{14}(1,3 \%)$. No se pueden realizar comparaciones extensas con respecto a la incidencia del tipo de infección ya que muy pocos autores las especifican. En este estudio, el porcentaje de urocultivos positivos es significativamente alto (95\%), superior a lo descrito en otras series, eso podría ser explicado en parte porque en nuestra institución las muestras de orina provenientes de pacientes con antecedente de uso de antibióticos se siembran en botellas de hemocultivos con resina lo que permite un $50 \%$ más de recuperación según estadísticas del hospital.

La frecuencia de Gram negativos en este estudio (90\%), fue superior a lo descrito en otras series, donde el porcentaje es cercano al $60 \%$, eso podría obedecer a las tendencias de la epidemiología local. ${ }^{14}$ Sin embargo, el Proteus mirabilis clásicamente descrito en asociación con cálculos coraliformes, solo se identificó en el $11 \%$ de los casos.

La mayoría de las infecciones se presentaron en pacientes del género femenino, evento contrario a lo descrito en la mayoría de los estudios, sin embargo, algunos autores como Kumar y colaboradores han encontrado que esa variable es un factor de riesgo independiente para el desarrollo de sepsis ${ }^{17}$

El 71\% de los pacientes se encontró el antecedente de Infección del Tracto Urinario en los 3 meses anteriores al procedimiento, eso constituye un factor de riesgo para desarrollar una complicación infecciosa descrita por diferentes autores $^{15}$

Si bien nuestra serie encontró un 38\% con hipertensión arterial como comorbilidad, ella no ha sido descrita como factor de riesgo de infección.

La mayoría de los cálculos presentaron un tamaño $25 \mathrm{~mm}$ (86\%), resultados que no discrepan con las indicaciones de la cirugía, esa variable ha sido identificada como factor de riesgo independiente para una complicación infecciosa ${ }^{18}$

Los pacientes en este estudio desarrollaron Infección urinaria pese a haber recibido profilaxis de antibiótico y aunque existe controversia en cuanto en qué momento iniciarla, su duración, y a que no elimina el riesgo de presentar complicaciones infecciosas, es una medida de prevención que se debe mantener, ajustando el antibiótico de acuerdo a la epidemiología local.

En dos tercios de los pacientes se debió escalar la cobertura de antibiótico pese a tener urocultivos, lo que resalta que los urocultivos vesicales previos no son buenos predictores del microorganismo que finalmente se relaciona con la UTI y de acuerdo a diferentes autores, Shoshany y col., el mejor predictor del agente etiológico es el cultivo de los fragmentos de cálculo ${ }^{13,16,19}$ 
Se encuentran muy pocos estudios que describen específicamente el tipo de complicación infecciosa que presentan los pacientes posteriormente a la NLP, en nuestro conocimiento, este es el primero que sería publicado proveniente de Latinoamérica.

Las debilidades del estudio son su naturaleza retrospectiva y descriptiva, que no permite establecer factores de riesgo independientes para infección.

\section{Conclusiones}

La NLP es el procedimiento de elección para el manejo de los cálculos de gran tamaño. Si bien el porcentaje de complicaciones infecciosas es bajo, esa complicación se traduce en mayor morbilidad para el paciente y aumento en los costos de la atención. Las infecciones post operatorias en este procedimiento se relacionan con pacientes con infecciones previas del tracto urinario en los 3 meses anteriores al procedimiento quirúrgico, pacientes cuyo tamaño de cálculo sea superior a $2,5 \mathrm{~cm}$ y su dureza supere las 1000 unidades Hounsfield. La adecuada valoración de los factores de riesgo individuales, la instauración de profilaxis adecuada y ajustada a la epidemiología local y el cultivo de los cálculos permite disminuir el riesgo de esa complicación y seleccionar la mejor opción terapéutica para los pacientes.

\section{Responsabilidades Éticas}

Protección de personas y animales Los autores declaran que para esta investigación no se han realizado experimentos en seres humanos ni en animales.

Confidencialidad de los datos Los autores declaran que en este artículo no aparecen datos de pacientes.

Derecho a la privacidad y consentimiento informado.

Los autores declaran que en este artículo no aparecen datos de pacientes.

En este artículo no aparecen datos que permitan identificar los pacientes.

Conflictos de Interés

Los autores declaran no tener conflictos de interés en relación a este estudio.

\section{Bibliografía}

1 Koras O, Bozkurt IH, Yonguc T, et al. Risk factors for postoperative infectious complications following percutaneous nephrolithotomy: a prospective clinical study. Urolithiasis 2015;43(01):55-60
2 Turk C, Knoll T, Petrik A, Sarica K, Straub M, Seitz C. Guidelines on urolithiasis. . Eur Assoc Urol. 2010:1-106

3 Dindo D, Demartines N, Clavien PA. Classification of surgical complications: a new proposal with evaluation in a cohort of 6336 patients and results of a survey. Ann Surg 2004;240(02):205-213

4 Tefekli A, Ali Karadag M, Tepeler K, et al. Classification of percutaneous nephrolithotomy complications using the modified clavien grading system: looking for a standard. Eur Urol 2008;53 (01):184-190

5 Negrete-Pulido O, Gutierrez-Aceves J. Management of infectious complications in percutaneous nephrolithotomy. J Endourol 2009;23(10):1757-1762

6 Margaret S, Pearle YL. Urinary Lithiasis Etiology, Epidemiology, and Pathogenesis. Chapter 45. 10 th edn. Elsevier; 2012

7 Brown PD. Management of urinary tract infections associated with nephrolithiasis. Curr Infect Dis Rep 2010;12(06):450-454

8 Skolarikos A, de la Rosette J. Prevention and treatment of complications following percutaneous nephrolithotomy. Curr Opin Urol 2008;18(02):229-234

9 Michel MS, Trojan L, Rassweiler JJ. Complications in percutaneous nephrolithotomy. Eur Urol 2007;51(04):899-906, discussion 906

10 Horan TC, Andrus M, Dudeck MA. CDC/NHSN surveillance definition of health care-associated infection and criteria for specific types of infections in the acute care setting. Am J Infect Control 2008;36(05):309-332

11 Salvadó B, José A, Astroza E. Gastón; Souper M., Renato. Complicaciones en nefrolitotomía percutánea. Rev Chil Urol 2014;79(01):45-50

12 Lee WJ, Smith AD, Cubelli V, et al. Complications of percutaneous nephrolithotomy. AJR Am J Roentgenol 1987;148(01):177-180

13 Dogan HS, Guliyev F, Cetinkaya YS, Sofikerim M, Ozden E, Sahin A. Importance of microbiological evaluation in management of infectious complications following percutaneous nephrolithotomy. Int Urol Nephrol 2007;39(03):737-742

14 Tefekli A, Ali Karadag M, Tepeler K, et al. Classification of percutaneous nephrolithotomy complications using the modified clavien grading system: looking for a standard. Eur Urol 2008;53 (01):184-190

15 Gutierrez J, Smith A, Geavlete P, et al; CROES PCNL Study Group. Urinary tract infections and post-operative fever in percutaneous nephrolithotomy. World J Urol 2013;31(05):1135-1140

16 Shoshany O, Margel D, Finz C, et al. Percutaneous nephrolithotomy for infection stones: what is the risk for postoperative sepsis? A retrospective cohort study. Urolithiasis 2015;43(03):237-242

17 Kumar S, Bag S, Ganesamoni R, Mandal AK, Taneja N, Singh SK. Risk factors for urosepsis following percutaneous nephrolithotomy: role of 1 week of nitrofurantoin in reducing the risk of urosepsis. Urol Res 2012;40(01):79-86

18 Mariappan P, Smith G, Bariol SV, Moussa SA, Tolley DA. Stone and pelvic urine culture and sensitivity are better than bladder urine as predictors of urosepsis following percutaneous nephrolithotomy: a prospective clinical study. J Urol 2005;173(05):1610-1614

19 Roushani A, Falahatkar S, Sharifi SH, Mahfoozi L, Saadat SM, Allahkhah A, Herfeh NR, Moghaddam KG. Intra-operative stone culture as an independent predictor of systemic inflammatory response syndrome after percutaneous nephrolithotomy. 